\title{
Polypoidal Choroidal Vasculopathy after Stereotactic Radiotherapy for Neovascular Age-Related Macular Degeneration: A Case Report with Long-Term Follow-Up
}

\author{
Alexandra Steinemann ${ }^{a} \quad$ Laura Hoffmann $^{a} \quad K^{2}$ atja B. Hatz ${ }^{a}$ b \\ ${ }^{a}$ Medical Retina Department, Vista Klinik Binningen, Binningen, Switzerland; ${ }^{b}$ Faculty of \\ Medicine, University of Basel, Basel, Switzerland
}

\section{Keywords}

Polypoidal choroidal vasculopathy · Stereotactic radiotherapy · Age-related macular degeneration

\begin{abstract}
Several adjunct therapies to the gold standard anti-vascular endothelial growth factor (antiVEGF) intravitreal injections have been discussed for the treatment of neovascular age-related macular degeneration (nAMD). Low-dose stereotactic radiotherapy (SRT) showed the potential to lower the treatment burden by reducing the anti-VEGF treatment frequency at least over 2-3 years but was associated with retinal microvascular abnormalities in a few cases. We report a 6-year follow-up of a case with bilateral nAMD under anti-VEGF treatment which developed multiple polypoid choroidal vasculopathy (PCV) lesions in the eye adjunct treated with low-dose SRT. The fellow eye suffering from nAMD for the same period of time but never been treated with SRT did not show PCV during the long-term follow-up. We hope to increase the awareness of possible choroidal changes such as PCV in similar patients by sharing this report.
\end{abstract}

\section{Introduction}

Neovascular age-related macular degeneration (nAMD) is one of the leading diseases of elderly people in industrialized countries accompanied by distinct loss of visual acuity (VA). The gold standard treatment for nAMD are intravitreal injections with anti-vascular endothelial growth factor (anti-VEGF) drugs, nowadays frequently applied in a treat-and-extend 
regime [1]. Frequent follow-ups including anti-VEGF injections is related with both great treatment burden and healthcare costs. Several adjunct therapies, such as photodynamic therapy and radiation therapy, have been discussed to prolong the anti-VEGF injection interval and/or reach a better treatment response [2-4]. Stereotactic radiotherapy (SRT) uses multiple narrow beams of radiation which are targeted to a small well-defined area with high precision. The randomized double-masked INTREPID study compared adjuvant SRT with SRT-sham treatment in anti-VEGF-treated nAMD [5]. The SRT group showed a reduction of anti-VEGF treatment during the 2 years of observation [5]. The same clinical outcome was shown in a 12-month real-life analysis [6]. However, a follow-up of the INTREPID trial reported retinal microvascular abnormalities (MVA) in about $30 \%$ of eyes, 3 years after SRT [7]. Retinal MVA found in color fundus photography and/or fluorescein angiography included nerve fiber infarcts, retinal hemorrhages, microaneurysms, luminal irregularities, focal areas of capillary closure, distended (bullous) arteriolar and venular tips, telangiectatic segments, and late staining of the vessel walls [7]. A reading center-based evaluation of real-life data confirmed these findings for clinical routine, too [8]. Yet, to the best of our knowledge, there are no reports on choroidal changes after adjunct SRT in nAMD so far.

\section{Case Report/Case Presentation}

A 69-year-old Caucasian male was referred by a local ophthalmologist with suspicion of bilateral nAMD in June 2014. Baseline diagnostic included VA and intraocular pressure measurement, biomicroscopic fundus examination, spectral-domain optical coherence tomography (SD-OCT; Spectralis, Heidelberg Eng., Heidelberg, Germany) with macular volume scan setting at high-speed mode, $20^{\circ} \times 20^{\circ}, 19$ sections ( 15 frames), macular star scan at high-speed mode, 6 sections ( 15 frames) and a 6-mm horizontal scan centered to the fovea; fluoresceine angiography with $30^{\circ}$ setting centered to the fovea (HRA2; Heidelberg Eng., Heidelberg, Germany) and indocyanine green angiography (ICG) with $30^{\circ}$ setting centered to the fovea (HRA2; Heidelberg Eng., Heidelberg, Germany). Corrected distance VA was 20/40 in the right and 20/32 in the left eye. In both eyes, the performed investigations revealed type 1 neovascularization (see Fig. 1) without signs of polypoid choroidal vasculopathy (PCV) and intravitreal injections using aflibercept $2 \mathrm{mg}$ (Eylea ${ }^{\circledR}$, Bayer, Switzerland) were started following a treat-and-extend schema as described before [9]. Despite reduction of subretinal fluid in both eyes, a treatment interval extension beyond 4 weeks was not possible due to persisting activity during the first 6 treatments. Therefore, after 6 aflibercept administrations in both eyes, the therapy was switched to ranibizumab $0.5 \mathrm{mg}$ (Lucentis ${ }^{\circledR}$, Novartis, Switzerland). However, with ranibizumab injected every 4 weeks, there was still persisting subretinal fluid especially in the right eye (Fig. 2b). Therefore, in the right eye (VA pre SRT 20/32), an adjunct SRT was performed using the IRay ${ }^{\circledR}$ System (Oraya Therapeutics, Newark, CA, USA; now Carl Zeiss Meditec AG, Jena, Germany) in May 2015 (4 days prior to the 6th ranibizumab [=12th anti-VEGF] injection). This noninvasive system comprises a precisioncontrolled X-ray tube, a patient interface, an eye stabilizing device, an eye-tracking system, a graphical user interface, and software for treatment planning. The IRay ${ }^{\circledR}$ System [10] and the procedure in our setting have been described in full previously [6] and were approved for clinical use in Europe. Three sequential beams converged to the retina and delivering a 16-Gy dose to an area of $4 \mathrm{~mm}$ in diameter centered to the fovea were applied.

As soon as 1 month after adjunct SRT, subretinal fluid was already markedly reduced (see Fig. 2c; VA 20/25), 2 months after SRT there were no signs of sub- or intraretinal fluid in the right eye (see Fig. 2d; VA 20/25). Therefore, a treatment interval extension in 2-week steps was started and proceeded without recurrences up to the maximum interval of 12 weeks

\section{Karger's}



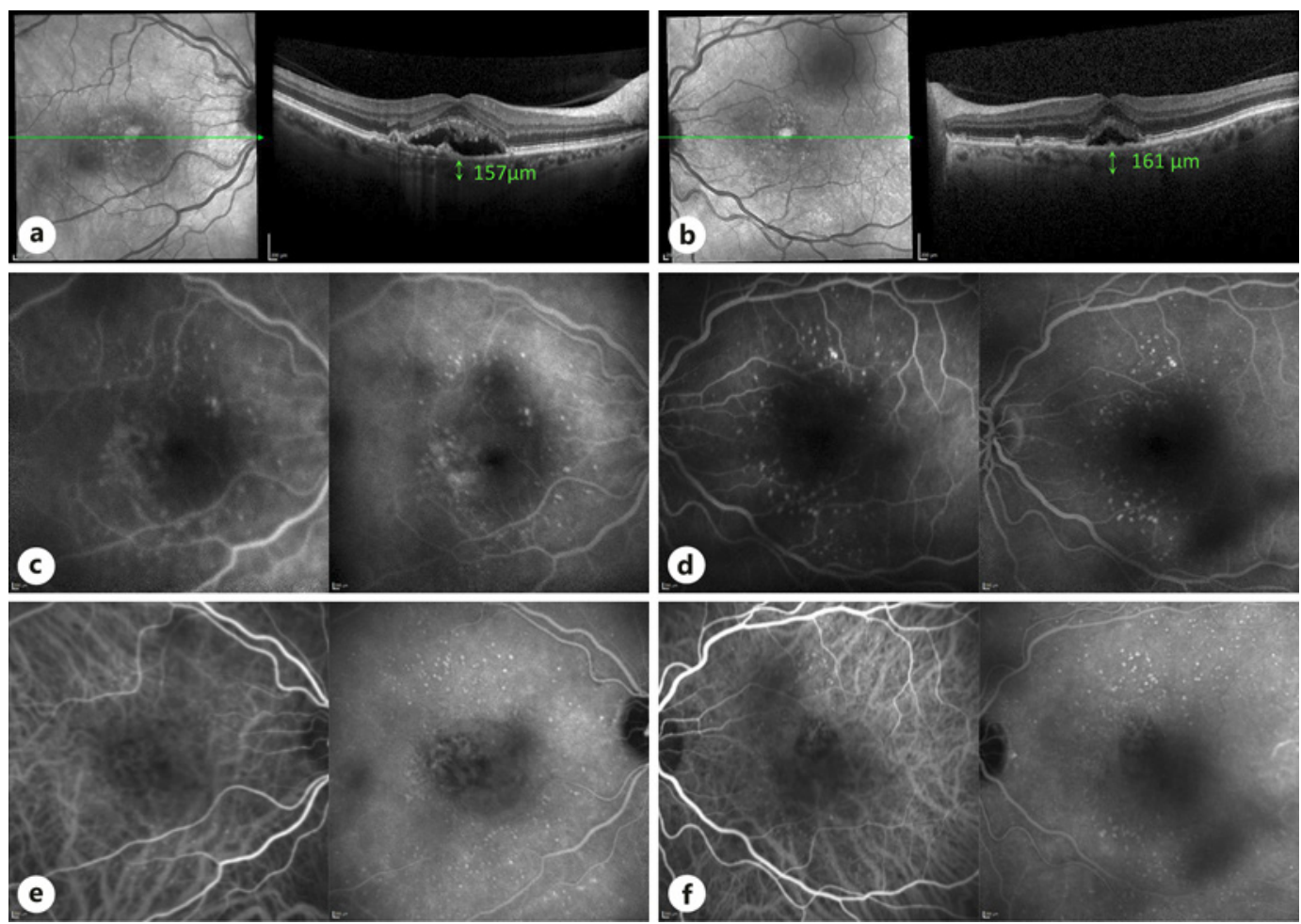

Fig. 1. Multimodal imaging of both eyes at baseline in July 2014. First row showing SD-OCT scans of the right (a) and left (b) eye with the corresponding central choroidal thickness (green arrows). Second row showing the FAG of the right (c) and left (d) eye in the early (left) and late (right) phases. ICG imaging of the right (e) and left (f) eye is shown in the last row with early (left) and late (right) phases. SD-OCT, spectral-domain optical coherence tomography; ICG, indocyanine green angiography.

(according to our treat-and-extend regimen [9]). Maintaining stability on treatment in a 12-week interval 3 consecutive times (last injection September 2016; VA 20/25), ranibizumab injections were stopped 16 months after SRT. Four weekly follow-up examinations including biomicroscopic fundus examination and SD-OCT scans in the right eye were continued in combination with treat-and-extend visits every 4 weeks for the left eye. In the left eye, the treatment was switched to aflibercept in December 2015 due to persisting subretinal fluid despite 4-week treatment intervals. An adjunct SRT in the left eye was not performed because of a lack of reimbursement by the health insurance. Twenty-one months after SRT and 5 months after stopping intravitreal ranibizumab injections, a first recurrence occurred in the right eye (see Fig. 2f; VA 20/25). Ranibizumab treatment was re-started with a 4-week treat-and-extend interval and led to a reduction but not complete resolution of subretinal fluid. After another 3 ranibizumab injections (total $17 \times$ ranibizumab, $6 \times$ aflibercept), the treatment in the right eye was re-switched to aflibercept in May 2017, 2 years after SRT. In the following period, both eyes were injected in a 4-week interval with only temporally dryness and a high risk of relapse after extension in the left eye and not reaching dryness in the right eye. VA remained stable between 20/25 and 20/20 (see online suppl. Table; for all online suppl. material, see www.karger.com/doi/10.1159/000514241) in both eyes. In the right eye, despite strict 4-week intervals, subretinal hemorrhages occurred in November 2019 and March 2020 (see Fig. 2i, j) with VA deteriorating to 20/50 in January 2020 but recovering to previous values of 20/25-20/20 under continued aflibercept therapy in March 2020. Due to COVID-19 pandemic, re-evaluation by fluoresceine and ICG was delayed up to June 2020. As shown in Figure 3 angiographies revealed a type 1 neovascularization pattern 
Steinemann et al.: Polypoidal Choroidal Vasculopathy after Stereotactic Radiotherapy for nAMD
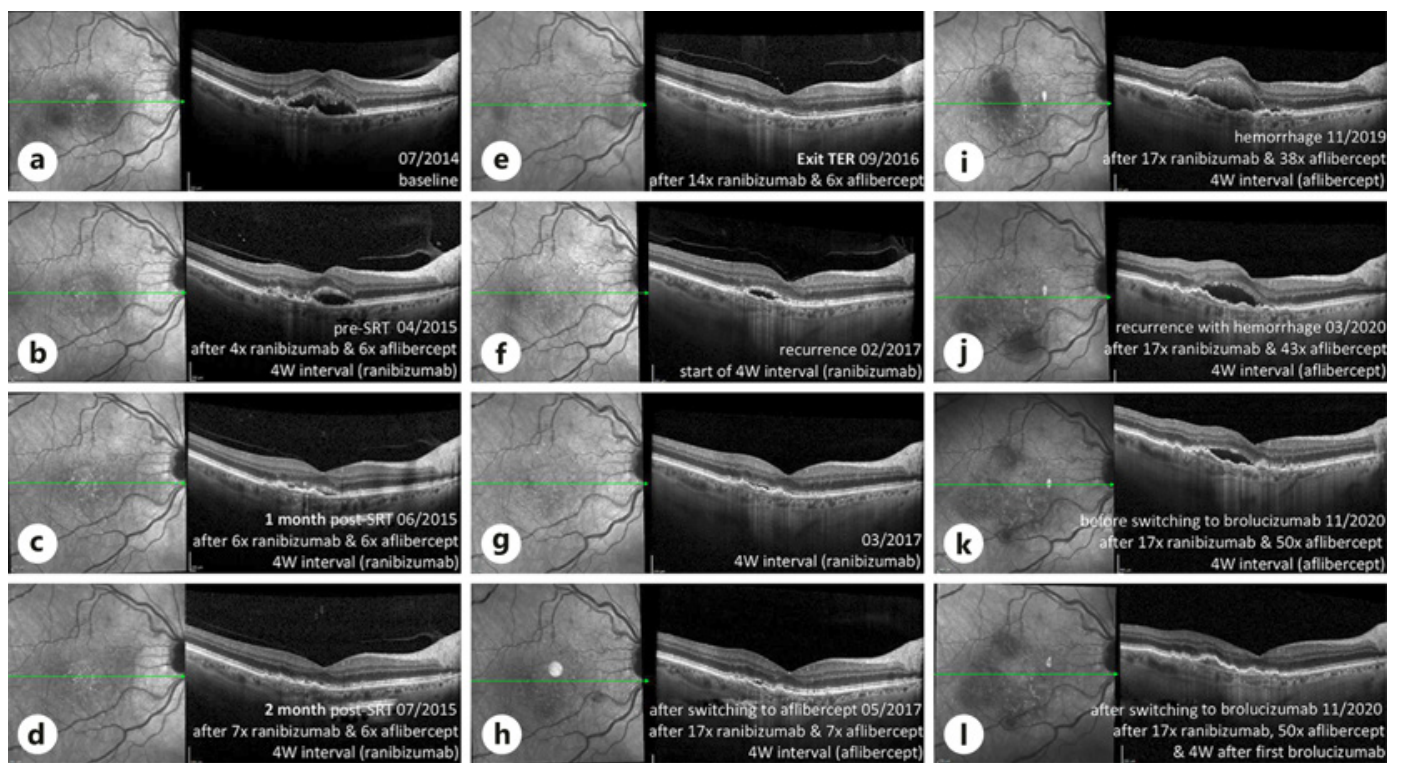

Fig. 2. Follow-up of SD-OCT scans of the right eye from baseline to November 2020. a Note the amount of subretinal fluid at baseline in July 2014 (VA 20/40). b Persistent subretinal fluid after 6 injections of aflibercept and 4 times ranibizumab in a 4-week interval (pre-SRT) in April 2015 with VA gain to 20/32. c One month post-SRT, the subretinal fluid was reduced, up to complete dryness 2 months post-SRT and VA improved to 20/25 which remained stable in the consecutive OCT scans (d). After the exit of the TER in September 2016 (e), a mild recurrence occurred in February 2017 (f). The subretinal fluid was initially responsive to ranibizumab (g) and later to aflibercept $(\mathbf{h})$. Despite strict intervals of 4 weeks with aflibercept, hemorrhages occurred in November 2019 (i) and March 2020, leading to a VA loss to 20/50 in January 2020 which recovered to 20/25 in March 2020 (j). Due to persistence of subretinal fluid, a therapy switch to brolucizumab was performed on 2 November 2020 (k) and resulted in decrease of subretinal fluid on 30 November 2020 (I). SD-OCT, spectral-domain optical coherence tomography; VA, visual acuity; TER, treat and extend regimen.

in both eyes with multiple polyps surrounding the neovascular network only in the right eye. Online suppl. Figure 1 shows the location of the type 1 choroidal neovascularization and the newly developed polyps in relation to the central 4-mm spot. In the right eye, central point choroidal thickness decreased from $157 \mu \mathrm{m}$ at baseline and before SRT to $103 \mu \mathrm{m}$ in June 2020 while it remained stable at about $160 \mu \mathrm{m}$ in the left eye (see online suppl. Table). Due to the partial atrophy of choroidea and possibly unknown alterations after SRT treatment, we did not consider to conduct a photodynamic therapy for the polyps. In the right eye, a switch to brolucizumab $6 \mathrm{mg}$ (Beovu ${ }^{\circledR}$, Novartis, Basel, Switzerland) was performed due to persisting subretinal fluid on 2 November 2020 (Fig. 2k). Four weeks later, subretinal fluid had resolved in almost all SD-OCT sections (Fig. 2l) and brolucizumab treatment was continued.

\section{Discussion}

For the current case, we report the extension of anti-VEGF treatment intervals during the first 2 years following adjunct SRT with stable VA. This corresponds to the findings of the INTREPID trial as well as a few real-life analyses [5, 6, 10-12]. However, like several patients in a real-life case series [8], our patient experienced the necessity of shortening of anti-VEGF treatment intervals in the third year after SRT. In both the INTREPID trial and the previous mentioned real-life analysis, SRT was associated with development of MVA occurring in the 
Steinemann et al.: Polypoidal Choroidal Vasculopathy after Stereotactic Radiotherapy for nAMD
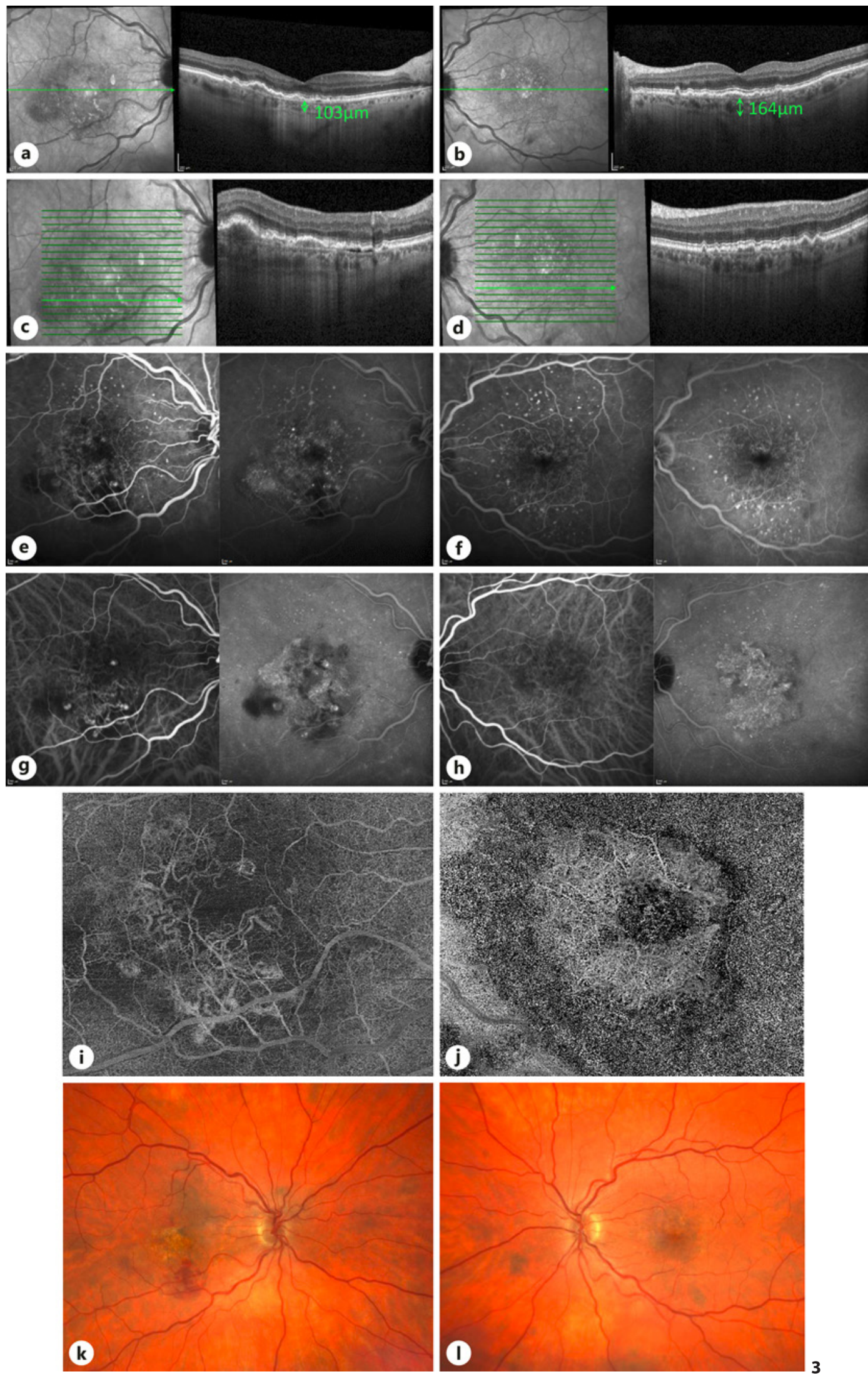

Karger' 
Fig. 3. Multimodal imaging of both eyes in June 2020. First 2 rows showing SD-OCT scans of the right (a, c) and left (b, d) eye with a "double-layer sign" in the right eye and decreased central choroidal thickness. Third row showing the FAG of the right (e) and left (f) eye in the early (left) and late (right) phases. $\mathbf{g}$ Note the presence of a single-foveal polyp and a cluster of polyps juxtafoveally inferiorly on ICG imaging of the right eye in the early (left) and late (right) phases. $\mathbf{h}$ The left eye shows an AMD-like neovascularization on ICG without PCV. Note the branching vascular network in the OCTA scan on the right (i) in contrast to the membrane on the left $(\mathbf{j})$. Color fundus photography reveals hemorrhages in the macular and juxtafoveal inferior area of the right eye (k) and no hemorrhage in the left eye (I). SD-OCT, spectral-domain optical coherence tomography; ICG, indocyanine green angiography; PCV, polypoid choroidal vasculopathy.

second and third year after SRT and being located most frequently in the parafoveal inferior and nasal region [7, 8]. While the parafoveal nasal appearance of more MVAs (mostly cotton wool spots) might be caused by the thicker retinal nerve fiber layer and its susceptibility to develop cotton wool spots the preference of the parafoveal inferior location remains unclear but was tried to be explained by the inferior part of the retina possibly being exposed to higher doses compared to the upper part due to the entrance of all 3 beams from the inferior sclera [8]. Our patient did not develop any of these retinal MVAs but choroidal changes such as multiple PCV lesions, most frequently located in the parafoveal inferior region, too.

Choroidal polyps were described and named "polypoidal choroidal vasculopathy" by Yannuzzi and colleagues [13]. While most PCV patients belong to Asian and African races, its prevalence in Caucasians should not be underestimated [14]. Initially, PCV was described as a distinct entity, but later also cases with features of nAMD were reported. In 2015, Coscas et al. [15] classified PCV into 2 types: the idiopathic variety and the nAMD type. The authors reported the nAMD type being associated with chorioretinal atrophy and the presence of a type 1 neovascularization. According to this classification and the absence of PCV at baseline, we assume our case belongs to the nAMD type. Our patient experienced a decrease in central choroidal thickness in the right but not in the left eye which suggests partial choroidal atrophy. Ranjbar et al. [11] reported the tendency to a decrease of the subfoveal choroidal thickness from baseline to 12 months after SRT. It remains unclear, whether the reported choroidal thinning in the right eye after SRT might have been associated with the development of nAMD type PCV [10]. It cannot be ruled out that SRT may not only be associated with MVA but also with polypoidal changes over the long-term. Therefore, it is important that SRT studies extend the follow-up so that SRT-treated patients are followed up on a regular basis in the long term.

\section{Conclusion}

Our case demonstrates the possibility of late choroidal changes such as PCV in patients previously treated with adjunct SRT for nAMD. Ophthalmologists should be aware of choroidal alterations as well as retinal MVA and follow SRT patients on a regular basis in the long term.

\section{Statement of Ethics}

Verbal and written informed consent was obtained from the patient for publication of this case report and all accompanying images.

\section{Conflict of Interest Statement}

The authors have no conflict of interest to declare.

\section{Karger'}




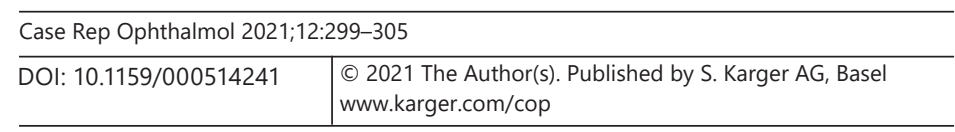

Steinemann et al.: Polypoidal Choroidal Vasculopathy after Stereotactic Radiotherapy for $\mathrm{nAMD}$

\author{
Funding Sources
}

Not applicable.

\title{
Author Contributions
}

A.S.: acquisition, interpretation of data, and writing of manuscript. L.H.: creation of figures and writing of manuscript. K.H.: supervision and writing of manuscript. All authors critically reviewed the manuscript and approved the final version of the manuscript.

\section{References}

1 Campbell JP, Bressler SB, Bressler NM. Impact of availability of anti-vascular endothelial growth factor therapy on visual impairment and blindness due to neovascular age-related macular degeneration. Arch Ophthalmol. 2012;130(6):794-5.

2 Evans JR, Sivagnanavel V, Chong V. Radiotherapy for neovascular age-related macular degeneration. Cochrane Database Syst Rev. 2010;(5):CD004004.

3 Gao Y, Yu T, Zhang Y, Dang G. Anti-VEGF monotherapy versus photodynamic therapy and anti-VEGF combination treatment for neovascular age-related macular degeneration: a meta-analysis. Invest Ophthalmol Vis Sci. 2018;59(10):4307-17.

4 Hatz K, Schneider U, Henrich PB, Braun B, Sacu S, Prünte C. Ranibizumab plus verteporfin photodynamic therapy in neovascular age-related macular degeneration: 12 months of retreatment and vision outcomes from a randomized study. Ophthalmologica. 2015;233(2):66-73.

5 Jackson TL, Chakravarthy U, Slakter JS, Muldrew A, Shusterman EM, O'Shaughnessy D, et al. Stereotactic radiotherapy for neovascular age-related macular degeneration: year 2 results of the INTREPID study. Ophthalmology. 2015;122(1):138-45.

6 Hatz K, Zimmermann F, Kardamakis D, Lazaridis E, Türksever C, Binder J, et al. Low-energy stereotactic radiotherapy for treatment of exudative age-related macular degeneration in a treat-and-extend regimen. Ophthalmic Surg Lasers Imaging Retina. 2018;49(2):86-93.

7 Freiberg FJ, Michels S, Muldrew A, Slakter J, O’Shaughnessy D, Czeszynski A, et al. Microvascular abnormalities secondary to radiation therapy in neovascular age-related macular degeneration: findings from the INTREPID clinical trial. Br J Ophthalmol. 2019;103(4):469-74.

8 Hatz K, Zimmermann F, Lazaridis E, Kardamakis D, Guichard M, Türksever C, et al. Microvascular abnormalities and long-term efficacy after stereotactic radiotherapy under continued intravitreal anti-VEGF treatment for neovascular AMD. Br J Ophthalmol. Forthcoming 2020.

9 Hatz K, Prünte C. Changing from a pro re nata treatment regimen to a treat and extend regimen with ranibizumab in neovascular age-related macular degeneration. Br J Ophthalmol. 2016;100(10):1341-5.

10 Jackson TL, Chakravarthy U, Kaiser PK, Slakter JS, Jan E, Bandello F, et al. Stereotactic radiotherapy for neovascular age-related macular degeneration: 52-week safety and efficacy results of the INTREPID study. Ophthalmology. 2013;120(9):1893-900.

11 Ranjbar M, Kurz M, Holzhey A, Melchert C, Rades D, Grisanti S. Stereotactic radiotherapy in neovascular agerelated macular degeneration: real-life efficacy and morphological evaluation of the outer retina-choroid complex. Medicine. 2016;95(52):e5729.

12 Brand C, Arnoldussen M. IRay therapy as an adjuvant therapy in newly diagnosed patients with neovascular age-related macular degeneration. Eye. 2018;32(8):1345-52.

13 Yannuzzi LA, Sorenson J, Spaide RF, Lipson B. Idiopathic polypoidal choroidal vasculopathy (IPCV). Retina. 1990;10(1):1-8.

14 Hatz K, Prünte C. Polypoidal choroidal vasculopathy in Caucasian patients with presumed neovascular agerelated macular degeneration and poor ranibizumab response. Br J Ophthalmol. 2014;98(2):188-94.

15 Coscas G, Lupidi M, Coscas F, Benjelloun F, Zerbib J, Dirani A, et al. Toward a specific classification of polypoidal choroidal vasculopathy: idiopathic disease or subtype of age-related macular degeneration. Invest Ophthalmol Vis Sci. 2015;56(5):3187-95. 